\title{
Mixing Characteristics of Binary Mixture with Biomass in a Gas-Solid Rectangular Fluidized Bed
}

\author{
Guiying Wu ${ }^{1}$, Bangting Yu ${ }^{2}$, Yanjun Guan ${ }^{1}$, Xuehui Wu ${ }^{1}$, Kai Zhang ${ }^{1, *}$ and Yongli Li ${ }^{1}$ \\ 1 Beijing Key Laboratory of Emission Surveillance and Control for Thermal Power Generation, \\ North China Electric Power University, Beijing 102206, China; wgy@cup.edu.cn (G.W.); \\ y.guan@ncepu.edu.cn (Y.G.); wuxhbj@126.com (X.W.); yongli.li@ncepu.edu.cn (Y.L.) \\ 2 CNOOC Research Institute, Beijing 100028, China; yubt3@cnooc.com \\ * Correspondence: kzhang@ncepu.edu.cn; Tel.: +86-10-6177-2413
}

Received: 18 April 2019; Accepted: 24 May 2019; Published: 26 May 2019

\begin{abstract}
Aiming to better understand the biomass pyrolysis and gasification processes, a detailed experimental study of the mixing characteristics is conducted in a fluidized bed with binary mixtures. Rapeseed is used as biomass, and silica sand or resin as inert material. The effect of mixture composition, initial packing manner, and superficial gas velocity on the concentration distribution is investigated in a rectangular fluidized bed by means of photography and sampling methods. The results show that the mixture composition plays an important role in the axial solids profile of binary mixtures. The mixing behavior of binary mixture is dominated by the bubble movement. The axial distribution of binary mixtures becomes uniform with increasing superficial gas velocity, whilst no obvious effect of initial packing manner is observed in this study.
\end{abstract}

Keywords: gas-solid fluidized bed; biomass mixture; concentration profile; mixing dynamics; bubble movement

\section{Introduction}

Gas-solid fluidized bed reactors have been extensively employed in biomass thermo-chemical conversion process, such as pyrolysis, gasification, combustion, and direct liquefication, because of their advantages of favorable gas-solid contact, high heat transfer coefficient, and uniform temperature profile [1-3]. Generally, an inert medium or catalyst is introduced as lubricant to facilitate the fluidization of biomass particles and to intensify the process transfer [4,5]. However, the mixing/segregation phenomenon of binary mixture results in complicated fluid dynamics within the fluidized bed when the inert medium or catalyst is added. In the past half century, the mixing and segregation have been regarded as two competing mechanisms to manipulate the particle distribution and flow pattern of binary mixture [6-9]. Rowe et al. [6] first proposed six possible binary combinations depending on the size and density of binary mixtures and found that segregation was particularly sensitive to density difference but less sensitive to size difference. Later, Nienow et al. [7] introduced the terms of "flotsam" and "jetsam" components to describe the particles, which occupy the top or bottom of the bed, respectively. Chiba et al. [8] suggested a general rule to distinguish the flotsam and jetsam components based on the findings of Rowe et al. [6] and Nienow et al. [7].

Solids mixing heavily depends on bubble movement in the fluidized beds [10-12]. Bubbles formed above the distributor carry particles in their wakes to the top of the bed. Upon reaching the surface, the bubble erupts and distributes particles. From the view of mass balance, particles in other regions of the bed move downward to compensate the upward flow of particles in the wake and drift of rising bubbles. Bubble wake particle transport mechanism and particle diffusion mechanism are two main types of models for quantifying the solids mixing in the fluidized bed [13]. Similarly, Mostoufi 
and Chaouki suggested the global and local patterns to describe the solids mixing in the fluidized bed [11]. However, limited work focused on the influence of bubble movement on solids mixing in the binary-particle system.

Accurate knowledge of the mixing/segregation phenomenon is fundamental in understanding hydrodynamic behavior containing biomass particles, which is becoming a challenge for thermo-chemical conversion processes of biomass in the fluidized bed reactor. In this study, the axial solids concentration profile of binary mixtures is investigated qualitatively and quantitatively in a gas-solids fluidized bed. The effect of mixture composition, initial packing manner, superficial gas velocity, and bubble movement on solids mixing is examined in detail.

\section{Experiments}

Biomass particles of rapeseed (RA) and bed material of silica sand (SS) or resin (RE) are used to establish binary systems with different volume fractions. Particle density is determined by Digital Density Meter (MDMDY-300, $\pm 0.15 \%$ ) and Electronic Balance (JT5003A, $\pm 0.001 \mathrm{~g}$ ), and bulk density is defined as the overall mass of materials per occupied volume measured in a calibrated cylinder. The minimum fluidization velocities of monodisperse particles and binary mixtures are investigated in a cylindrical fluidized bed with the inner diameter of $0.17 \mathrm{~m}$ and the height of $2.25 \mathrm{~m}$. Table 1 summarized the physical property of particles.

Table 1. Physical property of particles.

\begin{tabular}{cccc}
\hline Materials & Silica Sand (SS) & Resin (RE) & Rapeseed (RA) \\
\hline Geldart classification & $\mathrm{B}$ & $\mathrm{A}$ & $\mathrm{D}$ \\
Mean diameter $(d), \mathrm{mm}$ & 0.275 & 0.67 & 1.525 \\
Particle density $(\rho), \mathrm{kg} / \mathrm{m}^{3}$ & 2663 & 1448 & 1106 \\
Bulk density $\left(\rho_{\text {bulk }}\right), \mathrm{kg} / \mathrm{m}^{3}$ & 1250 & 814 & 595 \\
Minimum fluidization viodage $\left(\varepsilon_{\mathrm{mf}}\right)$ & 0.461 & 0.438 & 0.462 \\
Minimum fluidization velocity $\left(u_{\mathrm{mf}}\right), \mathrm{m} / \mathrm{s}$ & 0.11 & 0.207 & 0.490 \\
\hline
\end{tabular}

As illustrated in Figure 1, the mixing behavior of the binary mixture is examined qualitatively and quantitatively by using photography and sampling method in a pseudo two-dimensional (rectangular) fluidized bed with a cross-section area of $0.3 \times 0.025 \mathrm{~m}^{2}$ and a total height of $2.3 \mathrm{~m}$. The photography method employs a high-speed digital camera with the rate of 100 frames per second (AVT, PIKE F-032C). In this process a high intensity uniform illumination system is arranged around the bed and these instantaneous pictures are recorded for analysis.

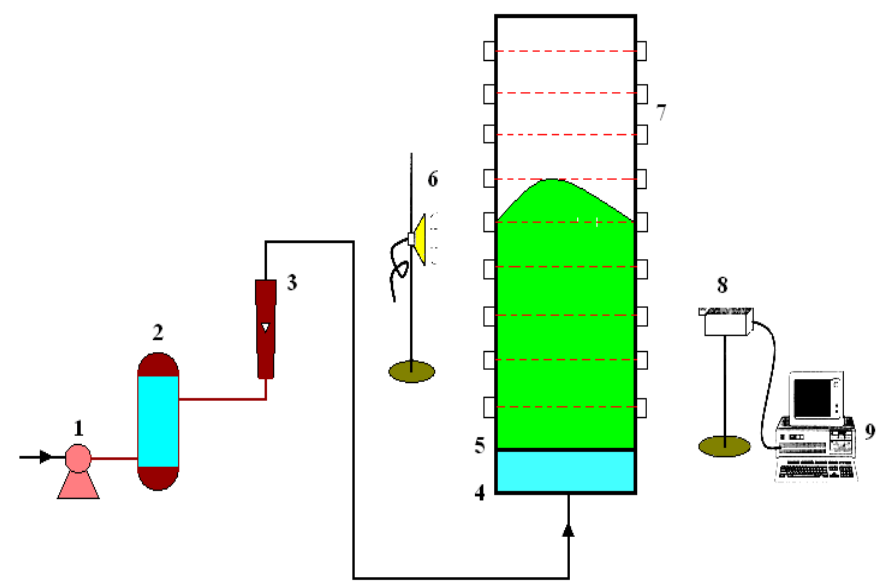

Figure 1. Schematic diagram of the experimental system. 1. Air compressor; 2. Tank; 3. Rotameter; 4. Plenum chamber; 5. Gas distributor; 6 . Light source; 7. Fluidized bed; 8 . High speed digital camera; 9. Computer. 
A stepwise sampling system designed in this study is shown in Figure 2. The lower part of the bed is equally divided into ten layers, and each layer has a height of $100 \mathrm{~mm}$ with a semicircular hole. In each experiment, the desired amount of biomass particle and inert material is first fed into the bed. Air from the compressor is measured by a rotameter and then introduced into the fluidized bed through the gas distributor. The fluidizing gas is slowly increased from zero to a desired value, and then suddenly shut off after a stable fluidization state is obtained. Subsequently, particles are withdrawn layer by layer from the 'frozen' bed. In order to obtain the axial concentration profile of inert medium or biomass, the particles removed from each section are sieved by a vibrating screen and then weighted by an Electronic Balance (LT2002) with a precision of $\pm 0.01 \mathrm{~g}$. More details about the sampling method can be found in the previous work [14]. Due to the uncertainty measurements of multiphase flow system, each case was repeated five times and the error was within $20 \%$ in this study, the averaged value was used to characterize the hydrodynamics. All experiments listed in Table 2 are conducted at room temperature and atmospheric pressure.

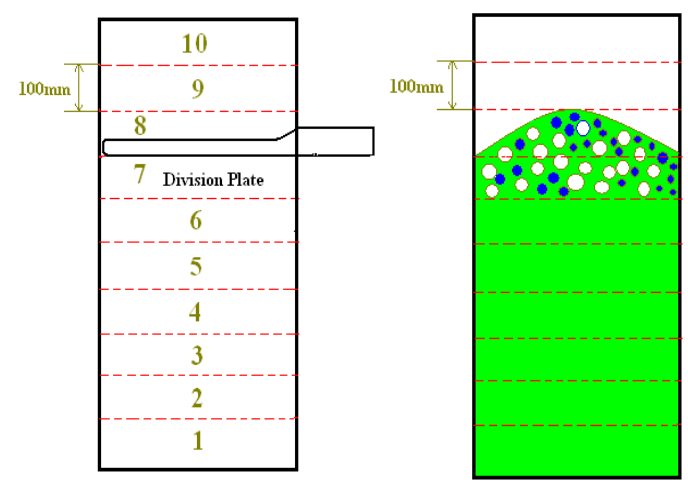

Figure 2. Schematic diagram of the stepwise sampling method.

Table 2. Experimental conditions.

\begin{tabular}{ccc}
\hline Binary System & RA-RE System & RA-SS System \\
\hline Initial packing conditions & RA on top & RA on top \\
& RA on bottom & RA on bottom \\
Complete mixed state & Complete mixed state \\
material $\left(V_{\mathrm{b}}\right)$ & $0.1,0.3,0.4,0.9$ & $0.1,0.3,0.5,0.9$ \\
Ratio of $u / u_{\mathrm{ff}}$ & $1.0 \sim 7.0$ & $1.0 \sim 6.0$ \\
\hline
\end{tabular}

\section{Results and Discussion}

\subsection{Fluidization Behaviors of Binary Systems}

Accurate knowledge of the minimum fluidization velocity is fundamental in understanding the hydrodynamic behavior of binary systems in the fluidized bed [15]. The general method of pressure drop vs. superficial gas velocity is employed to determine the minimum fluidization velocity in this section. Starting from the fixed-bed state, the superficial gas velocity is progressively increased until the bed reached a complete fluidization state and then is gradually decreased to zero. The interaction of the pressure drop line of the fixed-bed state with that for the fluidization state is denoted as the minimum fluidization condition, and the corresponding superficial gas velocity is defined as the minimum fluidization velocity. The minimum fluidization velocities of RA-SS and RA-RE with different volume ratios are examined in this work. As an example, Figure 3 illuminates the fluidization characteristics of the RA-RE binary mixture. It is clear that there are three characteristic fluidization velocities: the initial fluidization velocity $\left(u_{\mathrm{if}}\right)$, the minimum fluidization velocity $\left(u_{\mathrm{mf}}\right)$, and the full fluidization velocity $\left(u_{\mathrm{ff}}\right)$. The bed stays at the fixed-bed state when the superficial gas velocity is lower than $u_{\mathrm{if}}$, and the 
bed obtains the complete fluidized state when the superficial gas velocity is greater than $u_{\mathrm{ff}}$. The bed appears as the partial fluidized state when the superficial gas velocity is between $u_{\mathrm{if}}$ and $u_{\mathrm{ff}}$. Similar results are also obtained by Pei et al. [5] and Zhang et al. [16].

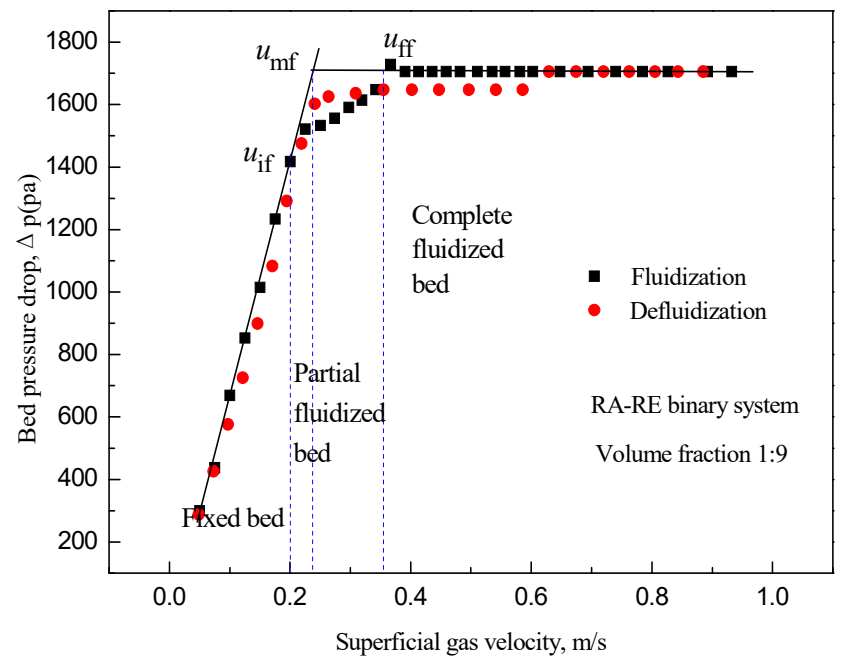

Figure 3. Characteristic fluidization velocities of the binary system $\left(V_{\mathrm{b}}=0.9\right)$.

\subsection{Effect of Mixture Composition on Concentration Distribution}

The effect of volume ratio on the solids mixing behavior of RA-SS and RA-RE is investigated quantitatively. The average concentration values of the inert medium are expressed as a dashed line in Figure 4 . For the RA-SS system operated at the fixed superficial gas velocity of $2.5 u_{\mathrm{ff}}$ as demonstrated in Figure 4a, the difference of SS concentration between the top and bottom layers increases with the increase in the initial volume fraction of jetsam component (SS), whilst their concentrations in the middle sections are close to the corresponding average value. Similar results are also obtained for the RA-RE system in Figure 4b.

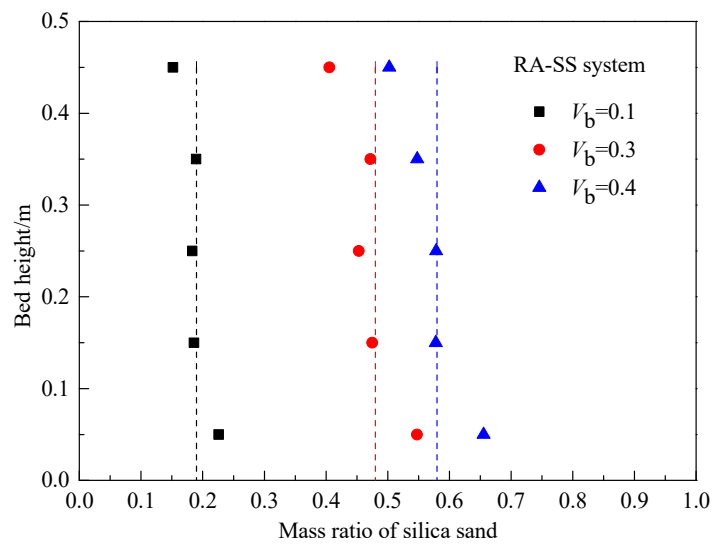

(a) RA-SS system $(2.5$ uff $)$.

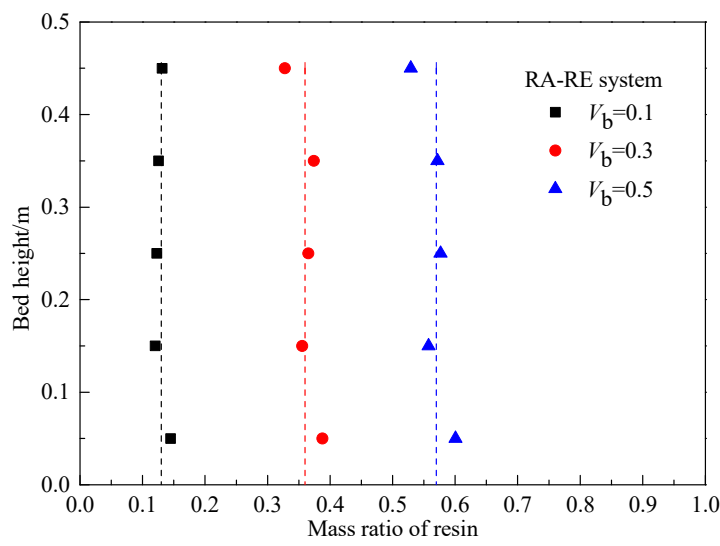

(b) RA-RE system (3.0 uff).

Figure 4. Effect of volume fraction on concentration profiles.

\subsection{Effect of Initial Packing Manners on Concentration Distribution}

Three initial packing conditions of the RA-SS system, including (a) RA on top, (b) RA on bottom, and (c) complete mixed state are investigated as shown in Figure 5. In order to achieve or approach the completely mixed state, the blends of binary mixture were homogenized by vigorous mixing with desired volume fractions and subsequently rolling for $10 \mathrm{~h}$ before the experiment. Frame-by-frame images show that three initial packing manners lead to different dynamic processes before the bed 
obtains the stable operation stage when the operating gas velocity is greater than the full fluidization velocity $\left(u_{\mathrm{ff}}\right)$. Figure 6 exhibits the quantitative results obtained by the stepwise sampling method when the operation has reached a stable stage. It is found that the mixing quality is improved by increasing the superficial gas velocity, whilst the influence of the packing conditions can be ignored for the binary mixtures used in this study. This phenomenon implies that the bubbles generated by high superficial gas velocity lead to noticeable turbulence in the bed although the initial packing manners are different.

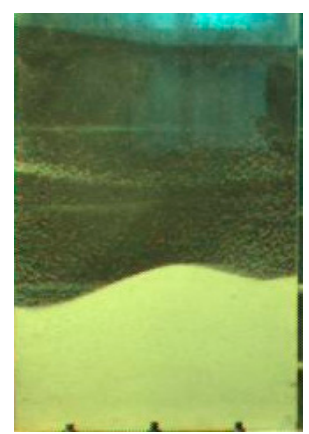

(a)

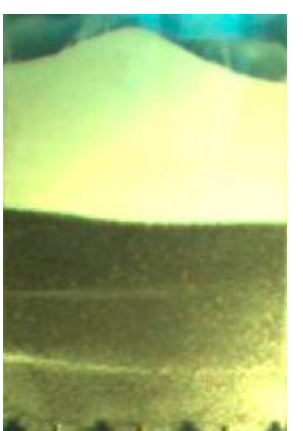

(b)

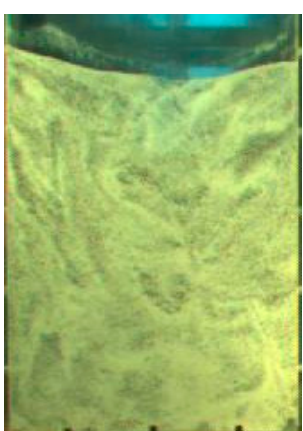

(c)

Figure 5. Three different initial packing conditions. (a) RA on top, (b) RA on bottom, and (c) complete mixed state.

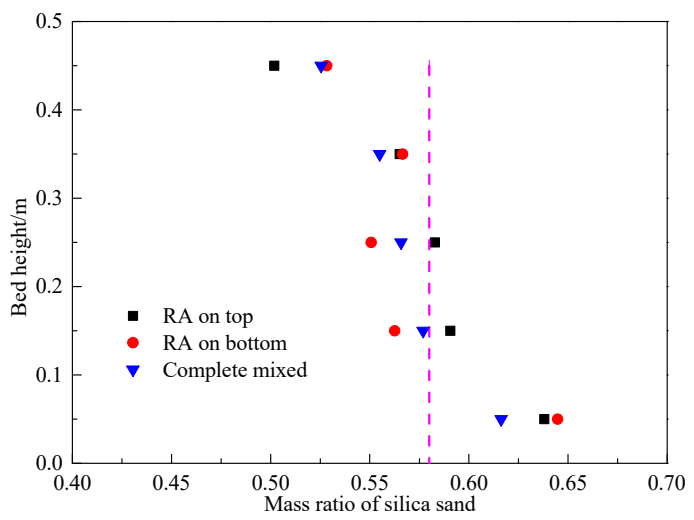

(a) $3.0 u \mathrm{ff}$

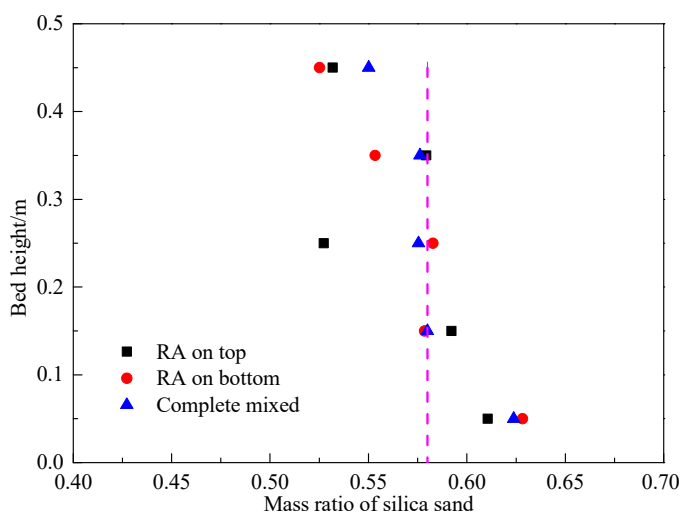

(b) $4.0 u$ ff.

Figure 6. Effect of initial packing conditions on SS concentration distribution.

\subsection{Effect of Superficial Gas Velocity on Concentration Distribution}

The effect of superficial gas velocity on the concentration profiles of RA-SS and RA-RE systems is examined qualitatively and quantitatively. Figure 7 exhibits that, qualitatively, bubble size increases with the increase of superficial gas velocity for both RA-SS and RA-RE systems. Furthermore, the quantitative distributions of inert medium in Figures 8 and 9 identify that the concentration difference of jetsam component (SS or RE) from the bottom to top in the bed decreases with the increase of superficial gas velocity, which is largely because of the strong turbulence induced by high gas velocity stirring particles vigorously through the fluidized bed. 


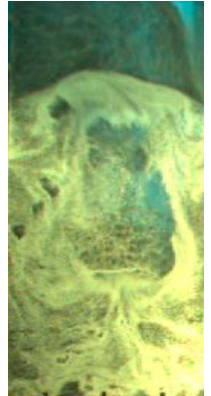

$2.0 u_{\mathrm{ff}}$

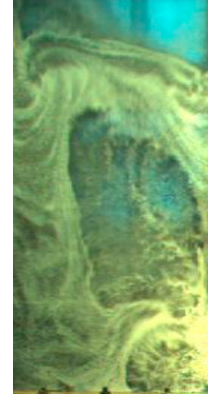

$2.5 u_{\mathrm{ff}}$

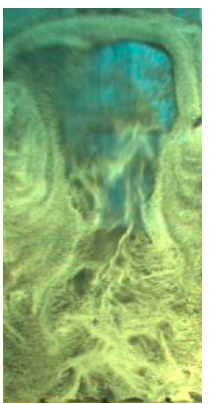

$3.5 u_{\mathrm{ff}}$

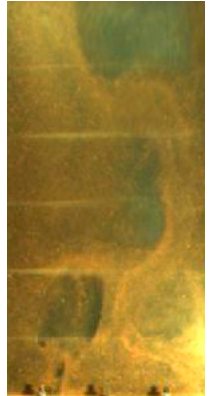

$2.5 u_{\mathrm{ff}}$

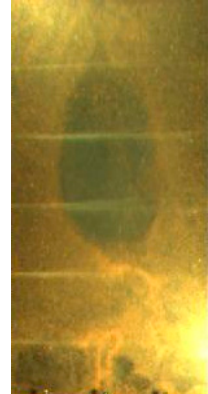

$3.0 u_{\mathrm{ff}}$

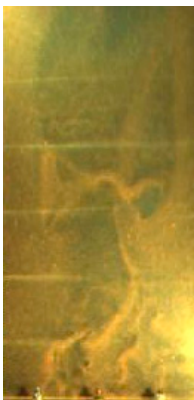

$4.0 u_{\mathrm{ff}}$

(a) RA-SS system.

(b) RA-RE system.

Figure 7. Qualitative mixing states of the binary mixtures $\left(V_{\mathrm{b}}=0.3\right)$.

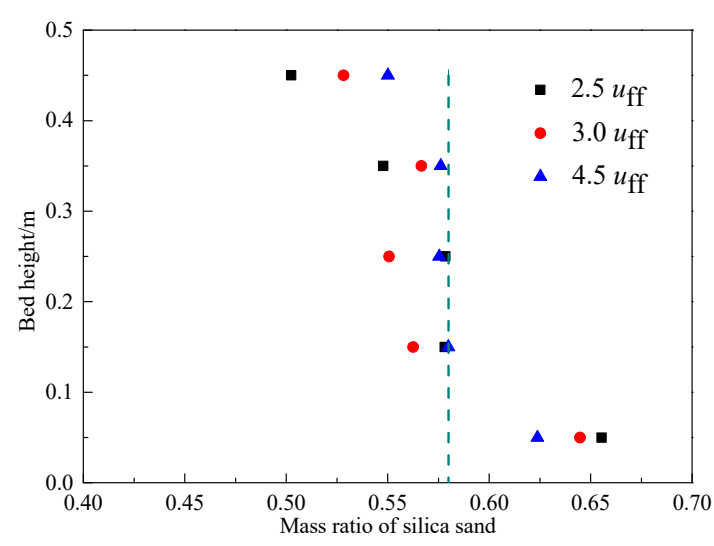

(a) $V_{\mathrm{b}}=0.4$.

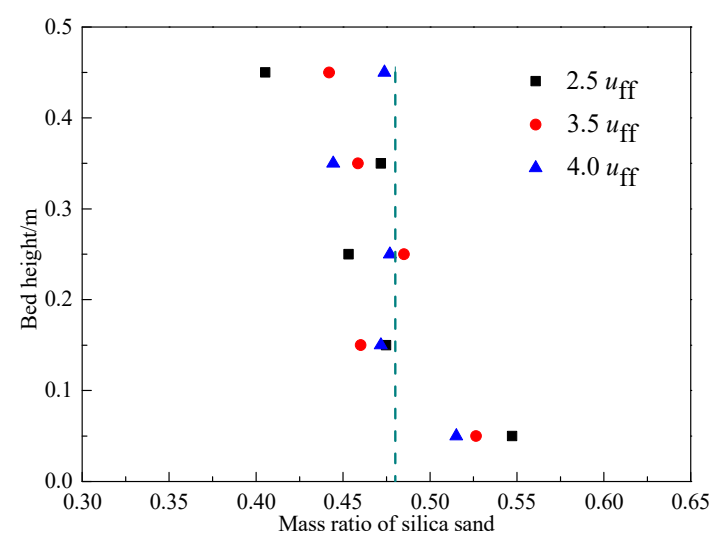

(b) $V_{\mathrm{b}}=0.3$.

Figure 8. Mass concentration profiles of SS in the RA-SS systems.

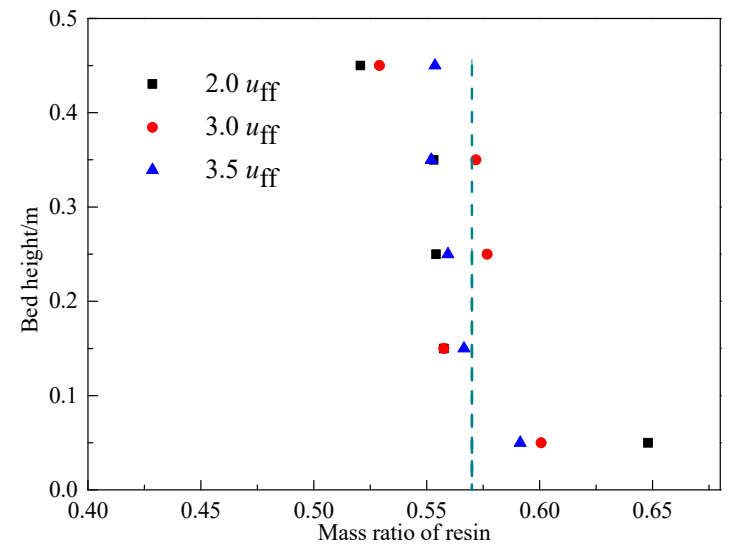

(a) $V_{b}=0.5$

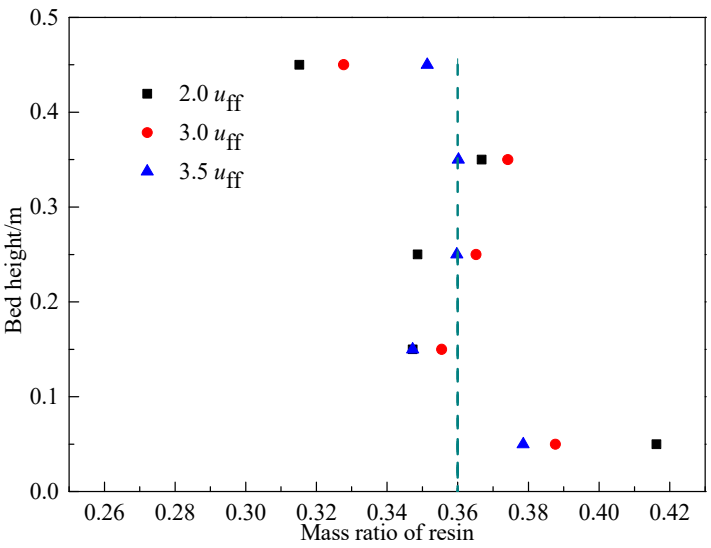

(b) $V_{b}=0.3$

Figure 9. Mass concentration profiles of RE in the RA-RE systems.

\subsection{Effect of Bubble Movement on Concentration Distribution}

The above investigations show that the mixing quality depends on superficial gas velocity more than the initial packing manner or mixture composition, which reveals that the bubble movement dominates the mixing/segregation phenomenon of binary mixture with biomass particles. Through analyzing the images captured by the digital video camera, solids mixing depends heavily on bubble movement for binary mixture, which is the same as that for the mono-particle system. On the whole, particles near to the wall region of the fluidized bed descend to supplement the cavities by the upstream motion. Meanwhile, particles slide along the periphery of a bubble and enter the wake of a bubble 
for a rising bubble. In order to understand the details, Figure 10 indicates that some particles in the roof of a bubble penetrate through the bubble to the wake region. This similar phenomenon was also found for the monodispersed particle systems by Rowe and Partridge [10] and Almendros-Ibáñez et al. [17] and for the binary mixtures by Bokkers et al. [18]. Rowe and Partridge [10] described this phenomenon as single bubbles were split in two by a "knife" of particles growing from the roof of the bubble. Almendros-Ibáñez et al. [17] regarded that particles fell from the dome of bubbles during their eruption process. Bokkers et al. [18] predicted the 'raining' of the particles through the roof of the bubble by discrete particle model.
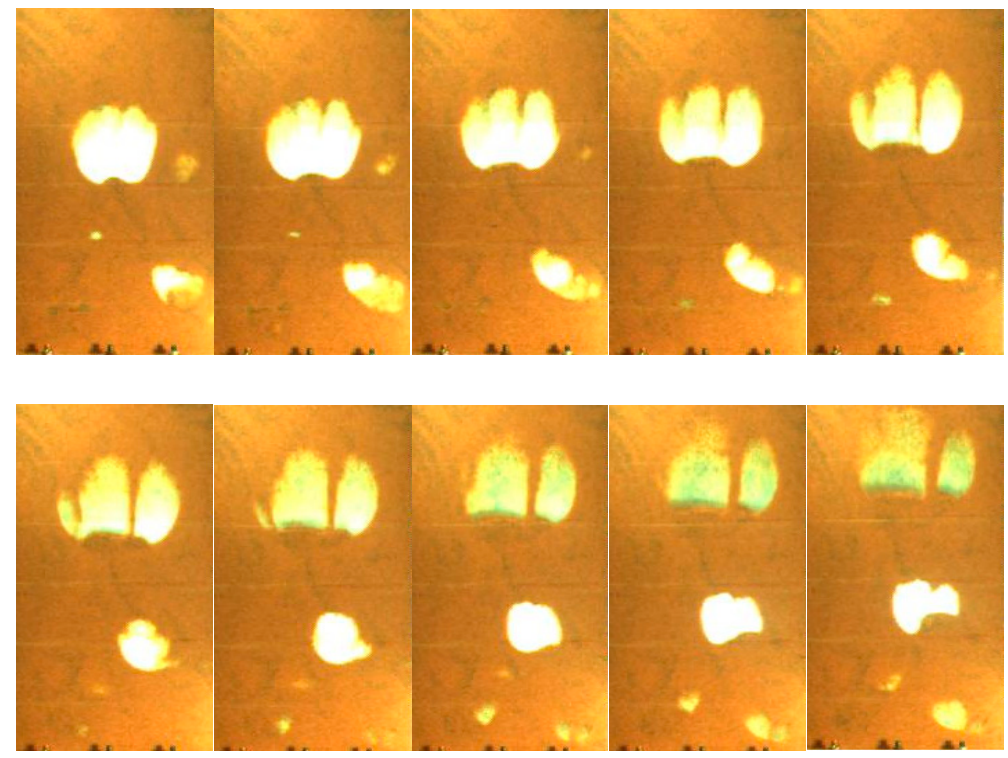

Figure 10. Descending processes of particles penetrated through bubble.

\section{Conclusions}

The mixing/segregation characteristics of binary mixtures with biomass are carried out in a gas-solid fluidized bed. The effects of the initial packing manner, mixture composition and superficial gas velocity on the solids mixing are investigated by photography and sampling methods. The following conclusions can be drawn from this work:

(1) Mixture composition has a distinct effect on the axial solids profile of binary mixtures. The difference of mass concentration between the top and bottom layers increases with increasing inert material (jetsam) fraction.

(2) Axial solids profile of binary mixtures becomes uniform with the increase of superficial gas velocity, whilst the influence of initial packing manner on the axial profile can be ignored when the superficial gas velocity is high enough.

(3) Solids mixing is dominated by the motion of bubbles. Particles move downward along the periphery of bubbles and near, but also penetrate the bubble.

Author Contributions: G.W. and B.Y. designed and performed the experiments, analyzed the date and wrote the paper; X.W. gave advise on the language logic; Y.G., K.Z. and Y.L. analyzed the data and provided the helpful discussion.

Funding: This research was funded by [National Natural Science Foundation of China-Shanxi coal based low carbon joint fund] grant number [U1610254] and [Creative Research Groups of the National Natural Science Foundation of China] grant number [51821004] and [111 Project] grant number [B12034] and [Fundamental Research Funds for the Central Universities] grant number [2018ZD03; 2018QN030].

Conflicts of Interest: The authors declare no conflict of interest. 


\section{References}

1. Cui, H.; Grace, J. Fluidization of biomass particles: A review of experimental multiphase flow aspects. Chem. Eng. Sci. 2007, 62, 45-55.

2. Mishra, R.K.; Mohanty, K. Pyrolysis characteristics and kinetic parameters assessment of three waste biomass. J. Renew. Sustain. Energy 2018, 10, 013102. [CrossRef]

3. Perez, N.; Pedroso, D.; Machin, E.; Antunes, J.; Ramos, R.; Silceira, J. Fluid dynamic study of mixtures of sugarcane bagasse and sand particles: Minimum fluidization velocity. Biomass Bioenerg 2017, 107, 135-149. [CrossRef]

4. Rao, T.; Bheemarasetti, J. Minimum fluidization velocities of mixtures of biomass and sands. Energy 2001, 26, 633-644. [CrossRef]

5. Pei, P.; Zhang, K.; Yu, B.; Gao, J.; Wu, G.; Wen, D. Dynamic characteristics of binary mixtures in a two-jet fluidized bed. Chem. Eng. Sci. 2011, 66, 1702-1714. [CrossRef]

6. Rowe, P.; Nienow, A.; Agbim, A. A preliminary quantitative study of particle segregation in gas fluidized beds binary systems of near spherical particles. Trans. Inst. Chem. Eng. 1972, 50, 324-333.

7. Nienow, A.; Rowe, P.; Cheung, L. A quantitative analysis of the mixing of two segregated powders of different density in a gas fluidised bed. Powder Technol. 1978, 20, 89-97. [CrossRef]

8. Chiba, S.; Nienow, A.; Chiba, T.; Kobayashi, H. Fluidized binary mixtures in which the denser component may be flotsam. Powder Technol. 1980, 26, 1-10. [CrossRef]

9. Manfred, W.; Franz, F.; Natalia, I.; Genadij, L. Particle mixing in bubbling fluidized beds of binary particle systems. Powder Technol. 2011, 120, 63-69.

10. Rowe, P.; Partridge, B. An X-ray study of bubbles in fluidised beds. Trans. Inst. Chem. Engrs. 1965, 43, 116-134. [CrossRef]

11. Mostoufi, N.; Chaouki, J. Local solid mixing in gas-solid fluidized beds. Powder Technol. 2001, 114, $23-31$. [CrossRef]

12. Wang, Y.; Cheng, Y.; Jin, Y.; Bi, H. On impacts of solid properties and operating conditions on the performance of gas-solid fluidization systems. Powder Technol. 2007, 172, 167-176. [CrossRef]

13. Kunii, D.; Levenspiel, O. Fluidization Engineering, 2nd ed.; Butterworth-Heinemann: Boston, MA, USA, 1991.

14. Zhang, K.; Yu, B.; Chang, J.; Wu, G.; Wang, T.; Wen, D. Hydrodynamics of a fluidized bed co-combustor for tobacco waste and coal. Bioresour. Technol. 2012, 119, 339-348. [CrossRef] [PubMed]

15. Perez, N.; Pedroso, D.; Machin, E.; Antunes, J.; Ramos, R.; Silceira, J. Prediction of the minimum fluidization velocity of particles of sugarcane bagasse. Biomass Bioenerg 2018, 109, 249-256. [CrossRef]

16. Zhang, Y.; Jin, B.; Zhong, W. Experimental investigation on mixing and segregation behavior of biomass particle in fluidized bed. Chem. Eng. Process. 2009, 48, 745-754. [CrossRef]

17. Almendros-Ibáñez, J.; Sobrino, C.; Vega, M.; Santana, D. A new model for ejected particle velocity from erupting bubbles in 2-D fluidized beds. Chem. Eng. Sci. 2006, 61, 5981-5990. [CrossRef]

18. Bokkers, G.; Annaland, M.; Kuipers, J. Mixing and segregation in a bidisperse gas-solid fluidised bed: A numerical and experimental study. Powder Technol. 2004, 140, 176-186. [CrossRef]

(C) 2019 by the authors. Licensee MDPI, Basel, Switzerland. This article is an open access article distributed under the terms and conditions of the Creative Commons Attribution (CC BY) license (http://creativecommons.org/licenses/by/4.0/). 